ESAIM: PROCEEDINGS, October 2007, Vol. 22, 162-168

Gabriel Caloz \& Monique Dauge, Editors

\title{
UNE MÉTHODE LEVELSET EN OPTIMISATION DE FORMES
}

\author{
Piotr Fulmanski ${ }^{1}$, Antoine Laurain ${ }^{2}$, Jean-Francois Scheid ${ }^{2,3}$ And Jan \\ SOKOLOWSKI ${ }^{2,4}$
}

\begin{abstract}
The topological derivative is a new tool introduced by Sokolowski and Zochowski in shape optimization. It allows to measure the variation of a functional depending on the geometrical domain when a small cavity is created inside the domain. It is possible to define the topological derivative for energy functionals of obstacle problems, and for contact problems without friction in solid mechanics. We present a few numerical results which confirm the validity of using the topological derivative in the framework of the levelset method for shape optimization of the Signorini problem.

Résumé. La dérivée topologique est un outil récent introduit par Sokolowski et Zochowski pour l'optimisation de formes. Elle permet de mesurer la variation d'une fonctionnelle dépendant d'un domaine géométrique quand on crée une petite cavité à l'intérieur de ce domaine. On peut définir la dérivée topologique pour les fonctionnelles d'énergie de problèmes d'obstacles, y compris les problèmes de contact sans frottement en mécanique des solides. Nous présentons quelques résultats, essentiellement numériques, qui confirment le bien-fondé de l'utilisation de la dérivée topologique dans le cadre d'une méthode levelset, pour l'optimisation de forme du problème de Signorini.
\end{abstract}

\section{INTRODUCTION}

Nous proposons une méthode numérique pour l'optimisation de formes d'une fonctionnelle de type énergie associée à un problème de Signorini. Cette méthode utilise la dérivée de forme et la dérivée topologique de la fonctionnelle. L'évolution géométrique des domaines est réalisée par une méthode de levelset (cf. [11], [12]). Les changements topologiques sont rendus possibles par l'utilisation de la dérivée topologique. La technique utilisée pour déterminer la dérivée topologique est basée sur les perturbations singulières de domaine (voir par exemple [9], [10], [13], [14]) et sur la construction de l'approximation asymptotique de l'opérateur de SteklovPoincaré (cf. [16]).

Commençons par décrire le problème modèle étudié. Soit $U$ et $V$ deux ouverts bornés de $\mathbb{R}^{2}$ tels que $V \subset \subset U$. Pour un ouvert $\omega$ de $\mathbb{R}^{2}$, on note $\# \bar{\omega}$ le nombre de composantes connexes de $\bar{\omega}$ et on considère l'ensemble des domaines admissibles

$$
\mathcal{O}_{k}=\{\Omega=U \backslash \bar{\omega} ; \omega \text { ouvert, } \omega \subset V, \# \bar{\omega} \leq k\}
$$

\footnotetext{
1 Universiti of Lodz, Faculty of Mathematics

Banacha 22, 90-232 Łódź, Poland ; e-mail: fulmanp@imul.math.uni.lodz.pl

2 Institut Elie Cartan, Laboratoire de Mathématiques, Université Henri Poincaré Nancy I,

B.P. 239, 54506 Vandoeuvre-lès-Nancy Cedex, France ; e-mail: antoine.laurain@iecn.u-nancy.fr

3 e-mail: jean-francois.scheid@iecn-u.nancy.fr

4 e-mail: sokolows@iecn.u-nancy.fr
} 
Pour $\Omega \in \mathcal{O}_{k}, k \geq 1$, on décompose le bord de $\Omega$ en $\partial \Omega=\Gamma_{N} \cup \partial U$ avec $\Gamma_{N}=\partial \omega$. Le bord $\partial U$ est séparé en deux composantes $\partial U=\Gamma_{S} \cup \Gamma_{D}$, la partie $\Gamma_{S}$ étant destinée à recevoir des conditions de Signorini et $\Gamma_{D}$ des conditions de Dirichlet. On va considérer deux problèmes d'optimisation de forme correspondants d'une part à un cas linéaire où seule une condition de Dirichlet est imposée sur $\partial U$ (c-à-d $\left.\Gamma_{S}=\emptyset\right)$ et d'autre part un cas non-linéaire avec une condition limite de Signorini $\left(\Gamma_{S} \neq \emptyset\right)$. Précisément, pour $f \in C^{\infty}(U)$, on considère d'une part le problème linéaire suivant :

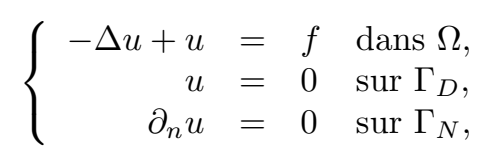

où $n$ désigne le vecteur unitaire normal à $\partial \Omega$ et dirigé vers l'extérieur de $\Omega$. Sans hypothèse supplémentaire sur la régularité de $\Omega$, on a $u \in H^{1}(\Omega)$. D'autre part, on considère le problème non-linéaire de Signorini

$$
\left\{\begin{aligned}
-\Delta u+u & =f \text { dans } \Omega, \\
u & =0 \text { sur } \Gamma_{D}, \\
\partial_{n} u & =0 \text { sur } \Gamma_{N}, \\
u \geq 0, \quad \partial_{n} u \geq 0, \quad u \partial_{n} u & =0 \text { sur } \Gamma_{S} .
\end{aligned}\right.
$$

La solution $u(\Omega)$ du problème (3) vérifie l'inéquation variationnelle

$$
u=u(\Omega) \in K(\Omega): \int_{\Omega} \nabla u \cdot \nabla(v-u) d x \geq \int_{\Omega}(f-u)(v-u) d x \quad \forall v \in K(\Omega),
$$

où

$$
K(\Omega)=\left\{v \in H^{1}(\Omega) \mid v=0 \text { p.p. sur } \Gamma_{D}, \quad v \geq 0 \text { p.p. sur } \Gamma_{S}\right\} .
$$

On considère maintenant les fonctionnelles suivantes.

$$
\begin{aligned}
E(\Omega, u) & =\frac{1}{2} \int_{\Omega}\left(|\nabla u|^{2}+u^{2}\right) d x-\int_{\Omega} f u d x \\
J(\Omega) & =E(\Omega, u)+\lambda A(\Omega)-\mu P_{c}(\Omega)^{2}
\end{aligned}
$$

avec $A(\Omega)$ et $P_{c}(\Omega)$ définis par

$$
\begin{aligned}
A(\Omega) & =|\Omega|, \\
P_{c}(\Omega) & =\max \left(0, \mathcal{H}^{1}(\partial \Omega)-c\right),
\end{aligned}
$$

où $|\Omega|$ est la mesure de Lebesgue de $\Omega$ dans $\mathbb{R}^{2}$ et $\mathcal{H}^{1}(\partial \Omega)$ est la mesure de Hausdorff 1 -dimensionnelle de $\partial \Omega$. Les constantes $\lambda, \mu$ sont strictement positives et introduisent respectivement des contraintes sur l'aire et le périmètre de l'ouvert $\Omega$. La constante $c$ est strictement positive et correspond à un périmètre seuil à partir duquel la contrainte de périmètre intervient. Pour $k \geq 1$, on s'intéresse alors au problème d'optimisation de forme suivant

$$
\max \left\{J(\Omega): \Omega \in \mathcal{O}_{k}\right\}
$$

associé successivement aux deux problèmes (2) et (3). On remarquera que si $u$ est la solution du problème linéaire (2) ou bien du problème de Signorini (3), alors l'énergie $E(\Omega, u)$ peut encore s'écrire

$$
E(\Omega, u)=-\frac{1}{2} \int_{\Omega} f u d x=-\frac{1}{2} \int_{\Omega}\left(|\nabla u|^{2}+u^{2}\right) d x .
$$




\section{LE PROBLÈME LINÉAIRE}

Analysons tout d'abord le problème d'optimisation (10) associé au problème linéaire (2).

\subsection{Existence d'un domaine optimal dans le cas linéaire}

On peut montrer l'existence d'un domaine $\Omega$ solution du problème (10) en utilisant un résultat de Bucur et Varchon [2]:

Theorem 1.1. Soient $\Omega=U \backslash \bar{\omega} \in \mathcal{O}_{k}, k \geq 1$ et $\Omega_{i}=U \backslash \bar{\omega}_{i}$ une suite d'ouverts telle \# $\bar{\omega}_{i} \leq k$. Si $\bar{\omega}_{i}$ converge au sens de la métrique de Hausdorff vers $\bar{\omega}$, alors la solution $u_{i}$ du problème de Neumann

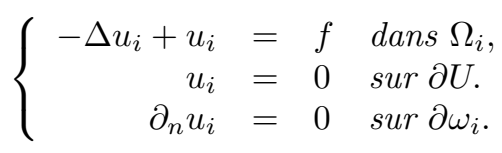

converge vers la solution $u$ du problème $(2)$ sur $\Omega$ si et seulement si $\left|\Omega_{i}\right| \rightarrow|\Omega|$. (Toutes les fonctions sont implicitement étendues par zéro en posant $u_{i}=0$ dans $\omega_{i}$ et $u=0$ dans $\omega$ et la convergence a lieu dans $\left.L^{2}(U)\right)$.

A partir du résultat précédent, on peut prouver l'existence d'un domaine optimal (voir [7]).

Theorem 1.2. Pour tout $k \geq 1$, le problème (10) associé à (2) admet au moins une solution $\Omega \in \mathcal{O}_{k}$.

\subsection{La dérivée par rapport au domaine dans le cas linéaire}

Soit $\xi$ un champ de vecteur régulier et à support compact dans $U$. Pour $t \geq 0$, on considère les transformations $F_{t}=I+t \xi$ et on note $\Omega_{t}=F_{t}(\Omega)$. Si $\Omega$ est suffisamment régulier, la fonctionnelle $J$ est dérivable par rapport au domaine (cf. [17]) et sa dérivée de forme définie par $d J(\Omega ; \xi)=\lim _{t \rightarrow 0}\left(J\left(\Omega_{t}\right)-J(\Omega)\right) / t$, est donnée par

$$
d J(\Omega ; \xi)=\int_{\Gamma_{N}}\left(\frac{1}{2}|\nabla u|^{2}+\frac{1}{2} u^{2}-f u+\lambda-2 P_{c}(\Omega) \mu \mathcal{H}\right)\langle\xi, n\rangle d \sigma
$$

où $\mathcal{H}$ est la courbure de $\Gamma_{N}$.

\subsection{La dérivée topologique pour le problème linéaire}

Pour calculer la dérivée topologique, on utilise une méthode appelée troncature de domaine et introduite en [8]. On introduit l'opérateur de Steklov-Poincaré qui dépend du rayon $\rho$ du trou, puis on réalise un développement asymptotique de cet opérateur par rapport à $\rho$.

On suppose pour simplifier que $\Omega=U$ c'est-à-dire que $\Omega$ n'a pas de bord intérieur $\left(\Gamma_{N}=\emptyset\right)$. Soit $x_{0} \in U$. On définit les domaines $\Omega_{\rho}$ et $\Omega_{R}$ par $\Omega_{\rho}=U \backslash \overline{B_{\rho}}, \Omega_{R}=U \backslash \overline{B_{R}}$ où $B_{\rho}$ et $B_{R}$ sont les boules centrées en $x_{0}$ et de rayons respectifs $\rho$ et $R$ avec $R>\rho$. Les bords de $B_{\rho}$ et $B_{R}$ sont notés respectivement $\Gamma_{\rho}=\partial B_{\rho}$ et $\Gamma_{R}=\partial B_{R}$. On définit alors le problème suivant

$$
\left\{\begin{array}{rrrrr}
-\Delta u_{\rho}+u_{\rho} & = & f & \text { dans } & \Omega_{\rho} \\
\partial_{n} u_{\rho} & = & 0 & \text { sur } & \Gamma_{\rho} \\
u_{\rho} & = & 0 & \text { sur } & \partial U
\end{array}\right.
$$


On veut calculer le développement asymptotique par rapport à $\rho$ de l'énergie $E\left(\Omega_{\rho}, u_{\rho}\right)=-\frac{1}{2} \int_{\Omega_{\rho}}\left(\left|\nabla u_{\rho}\right|^{2}+u_{\rho}^{2}\right) d x$ où $u_{\rho}$ est la solution de (14). La méthode de troncature de domaine consiste à introduire le problème suivant

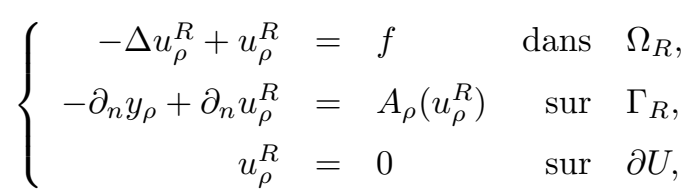

où $A_{\rho}$ est l'opérateur de Steklov-Poincaré défini par $A_{\rho}(v)=\partial_{n}\left(w_{\rho}\right)$ avec $w_{\rho}=w_{\rho}(v)$ la solution de

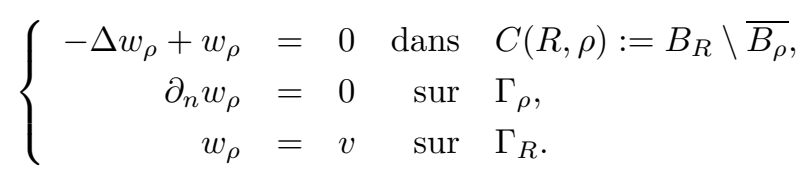

Dans le problème (15), la fonction $y_{\rho}$ est la solution du problème

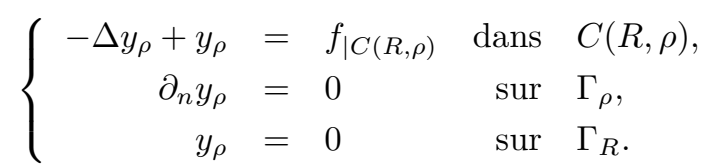

En utilisant le fait que $u_{\rho}^{R}=u_{\rho_{\mid \Omega_{R}}}$ et $u_{\rho \mid C(R, \rho)}=w_{\rho}\left(u_{\rho}^{R}\right)+y_{p}$ et en étudiant successivement les problèmes (15), (16) et (17), on obtient le développement asymptotique de l'énergie $E\left(\Omega_{\rho}, u_{\rho}\right)$ pour le problème (14) (cf. [7]):

$$
E\left(\Omega_{\rho}, u_{\rho}\right)=-\frac{1}{2} \int_{\Omega_{\rho}}\left(\left|\nabla u_{\rho}\right|^{2}+u_{\rho}^{2}\right) d x=E(\Omega, u)+\left[-\frac{u\left(x_{0}\right)^{2}}{2}-\left|\nabla u\left(x_{0}\right)\right|^{2}+u\left(x_{0}\right) f\left(x_{0}\right)\right] \pi \rho^{2}+o\left(\rho^{2}\right),
$$

où $u$ est la solution du problème linéaire (2). En tenant compte des développements de l'aire et du périmètre

$$
\begin{aligned}
A\left(\Omega_{\rho}\right) & =A(\Omega)-\pi \rho^{2}, \\
P_{c}\left(\Omega_{\rho}\right)^{2} & =P_{c}(\Omega)^{2}+4 \pi P_{c}(\Omega) \rho+\rho^{2},
\end{aligned}
$$

on en déduit que la dérivée topologique $\mathcal{T}_{\Omega}\left(x_{0}\right)$ de $J$ en $x_{0} \in \Omega$ est donnée par

$$
\begin{cases}\mathcal{T}_{\Omega}\left(x_{0}\right)=-\left|\nabla u\left(x_{0}\right)\right|^{2}-\frac{1}{2} u\left(x_{0}\right)^{2}+u\left(x_{0}\right) f\left(x_{0}\right)-\lambda & \text { si } P_{c}(\Omega)=0 \\ \mathcal{T}_{\Omega}\left(x_{0}\right)=-4 \mu P_{c}(\Omega) \pi & \text { si } P_{c}(\Omega)>0\end{cases}
$$

\section{LE PROBLÈME NON-LINÉAIRE DE SignORINI}

On s'intéresse à présent au problème non-linéaire (3) pour le problème d'optimisation (10).

\subsection{Dérivation par rapport au domaine pour le problème non-linéaire}

On montre que la dérivée de forme $d J$ existe dans le cas non-linéaire et qu'elle est donnée par la même relation (13) que dans le cas linéaire précédent. Toutefois, dans le cas non-linéaire, il s'agit seulement d'une dérivée directionnelle et non pas d'une dérivée au sens de Fréchet comme pour le problème linéaire.

\subsection{La dérivée topologique pour le problème non-linéaire}

On a utilisé pour le problème linéaire la méthode de troncation de domaine. L'intérêt de cette technique est qu'elle peut être utilisée également dans le cas non-linéaire du problème de Signorini. On en déduit la même formule (19) pour la dérivée topologique de $J$ que dans le cas linéaire (voir [4]). 


\section{LA FORMUlation "LEVElSET"}

\subsection{L'équation de Hamilton-Jacobi}

L'idée de base de la méthode levelset est de représenter un domaine et sa frontière comme des lignes de niveau d'une fonction continue $\phi$ définie sur tout le domaine $U$ (voir [11], [12]). Considérons la transformation du domaine $\Omega_{t}=(I+t \xi)(\Omega) \subset U \subset \mathbb{R}^{2}, t \in \mathbb{R}^{+}$, par un champ de vitesse $\xi$ régulier. Le domaine et la frontière sont définis par une fonction $\phi=\phi(x, t)$ telle que

$$
\Omega_{t}=\{x \in U, \phi(x, t)<0\} \quad \text { et } \quad \partial \Omega_{t}=\{x \in U, \phi(x, t)=0\} .
$$

L'évolution de $\phi$ est alors gouvernée par l'équation de Hamilton-Jacobi

$$
\phi_{t}+\xi_{n}|\nabla \phi|=0 \quad \text { dans } U \times \mathbb{R}^{+}
$$

où $\xi_{n}=\langle\xi, n\rangle$ est la vitesse normale et $n(x, t)=\nabla \phi(x, t) /|\nabla \phi(x, t)|$ est la normale à la courbe de niveau de $\phi$ passant par le point $x$. Compte tenu de l'expression (13) de la dérivée de forme de $J$, on fait le choix suivant pour la composante normale $\xi_{n}$ du champ de vitesse

$$
\xi_{n}=\frac{1}{2}|\nabla u|^{2}+\frac{1}{2} u^{2}-f u+\lambda-2 P_{c}(\Omega) \mu \mathcal{H} \quad \operatorname{sur} \Gamma_{N} .
$$

Pour un tel champ de vitesse $\xi$, on a alors clairement $d J(\Omega ; \xi) \geq 0$.

\section{Algorithme D’optimisation et résultats numériques}

Pour déterminer un ouvert optimal résolvant (10), on utilise la formulation "levelset" précédente et la dérivée topologique de $J$. On procède de la façon suivante. Tout d'abord, on calcule la solution du problème (2) ou (3) à l'aide d'une méthode d'éléments finis dans un domaine initial $\Omega^{(0)}$. On peut alors calculer la dérivée topologique (19) qui nous indique où créer un trou dans le domaine $\Omega^{(0)}$ : on cherche un point $x_{0} \in \Omega^{(0)}$ tel que $\mathcal{T}_{\Omega^{(0)}}\left(x_{0}\right)=\max _{x \in \Omega^{(0)}} \mathcal{T}_{\Omega^{(0)}}(x)$. Si $\mathcal{T}_{\Omega^{(0)}}\left(x_{0}\right)>0$ alors on crée un trou centré en $x_{0}$. Le rayon de ce trou doit être aussi petit que possible en fonction de la taille du maillage. On obtient ainsi un domaine $\Omega_{*}^{(0)}$. On procède ensuite à l'évolution du domaine $\Omega_{*}^{(0)}$. On doit calculer les solutions $\phi$ de l'équation d'Hamilton-Jacobi (21). La fonction $\phi$ initiale est prise comme la fonction distance signée au domaine $\Omega_{*}^{(0)}$. Comme la vitesse normale $\xi_{n}$ est connue seulement sur la frontière interne $\Gamma_{N}$ de $\partial \Omega^{0}$, on doit l'étendre à tout le domaine $U$ (cf. [4], [11], [12]). Une fois que la fonction levelset est calculée, on peut déterminer le nouveau domaine $\Omega^{(1)}$ et recommencer le procédé jusqu'à la convergence vers un ouvert optimal.

Pour le problème linéaire, on obtient des résultats numériques qui montrent l'intérêt de l'utilisation de la dérivée topologique. Avec les données $f(x, y)=10 \sin ^{2}(4 \pi x)$ pour $(x, y) \in \Omega^{(0)}, \lambda=0.5, \mu=0$, on réalise deux exemples. Dans le premier exemple, le domaine initial $\Omega^{(0)}=(0,1) \times(0,1)$ n'a pas de trou et on utilise la dérivée topologique. On obtient ainsi un domaine $\Omega_{1}$ (cf. Figure 1).

Dans le deuxième exemple, le domaine initial est le pavé $(0,1) \times(0,1)$ contenant 21 trous et la dérivée topologique n'est pas utilisée. On obtient dans ce cas un domaine $\Omega_{2}$ (cf. Figure 2). On constate que l'utilisation de la dérivée topologique dans le premier exemple permet une amélioration de $6.5 \%$ de la fonctionnelle $J$ par rapport au deuxième exemple qui n'utilise pas la dérivée topologique. En effet, on obtient

$$
J\left(\Omega_{2}\right)=0.2640695<J\left(\Omega_{1}\right)=0.282319
$$

Par ailleurs, l'utilisation de la dérivée topologique permet de s'affranchir d'un choix judicieux du domaine initial, souvent déterminé par une forme pressentie de l'ouvert optimal. 
$\Omega_{1}$ (331 itérations)
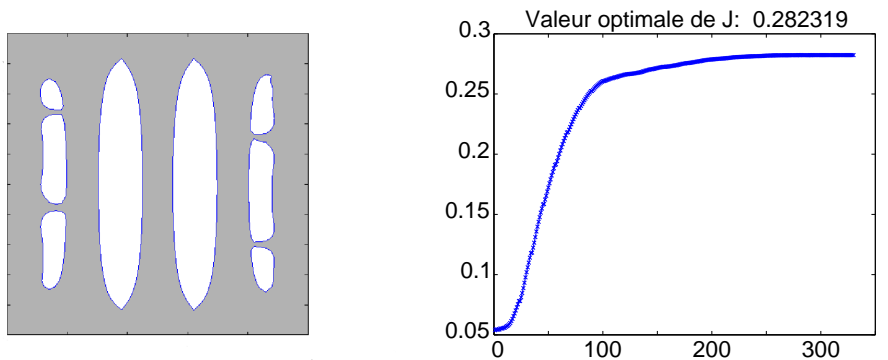

Figure 1. Domaine $\Omega_{1}$ obtenu après 331 itérations et fonctionnelle $J$ pour le cas linéaire avec le domaine initial $\Omega^{(0)}=(0,1) \times(0,1)$ et utilisation de la dérivée topologique.

Domaine initial

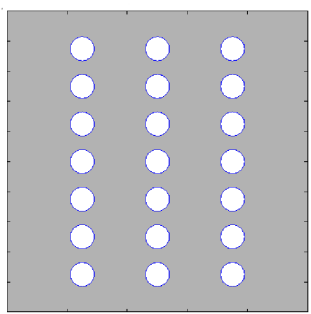

$\Omega_{2}$ (331 itérations)

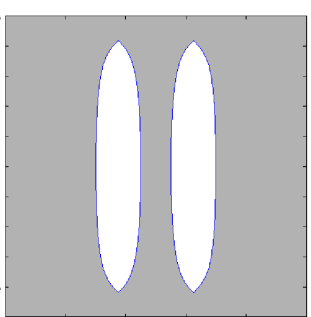

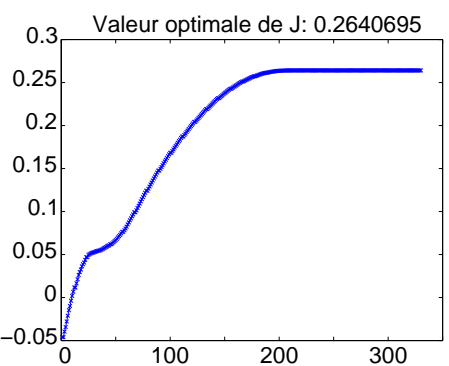

FIGURE 2. Domaine initial $\Omega^{(0)}$, domaine $\Omega_{2}$ obtenu après 331 itérations et fonctionnelle $J$ pour le cas linéaire sans utilisation de la dérivée topologique.

Dans le troisième exemple (voir Figure 3), on s'intéresse au cas non-linéaire avec $\lambda=0.3, \mu=0.001$ et $c=0.6$. La fonction source $f$ est a support compact dans $U$. On constate que la convergence vers un maximum a lieu globalement, mais localement, la fonctionnelle est beaucoup plus sensible au petites perturbations du domaine. Ceci s'explique par la nature différente des dérivées dans les problèmes linéaire et non-linéaire (dérivée au sens de Fréchet pour le problème linéaire et dérivée au sens de Gâteaux pour le problème non-linéaire).

\section{REFERENCES}

[1] G. Allaire, F. De Gournay, F. Jouve, A.M. Toader, Structural optimization using topological and shape sensitivity via a level set method, Control and cybernetics (2005).

[2] D. Bucur, N. VArchon, Boundary variation for a Neumann problem, Ann. Scuola Norm.Sup.Pisa Cl.Sci., 4 (2000), no. 29, pp. 807-821.

[3] M.C. Delfour, J.-P. Zolesio, Shapes and Geometries, Advances in Design and Control. Society for Industrial and Applied Mathematics (SIAM), Philadelphia, PA, 2001.

[4] P. Fulmanski, A. Laurain, J.-F. Scheid, J. Sokolowski, A levelset method in shape and topology optimization for variational inequalities, Prépublications Institut Elie Cartan, Université Nancy 1, 2006, no 32.

[5] A. Henrot, M. Pierre, Variation et optimisation de formes: une analyse géométrique, No 48 de Mathématiques et Applications, Springer, 2005.

[6] J. Jarusek, M. Krbec, M. Rao, J. Sokolowski, Conical differentiability for evolution variational inequalities, J. Differential Equations, 193, (2003), no. 1, pp. 131-146.

[7] A. Laurain, Domaines singulièrement perturbés en optimisation de formes, thèse de doctorat, Université de Nancy 1, 2006.

[8] M. Masmoudi, The topological asymptotic, in: H;Kawarada, J.Periaux (Eds.), Computationnal Methods for Control Applications, International Series GAKUTO, 2002.

[9] S.A. Nazarov, J. SokoŁowski, Asymptotic analysis of shape functionals Journal de Mathématiques pures et appliquées. 82 (2003), pp. 125-196. 
Domaine initial

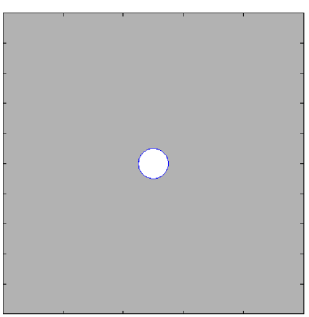

itération 55

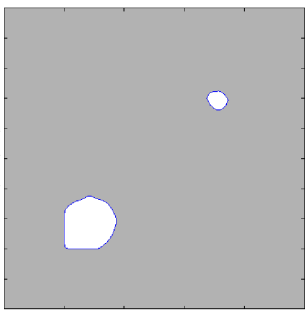

itération 9

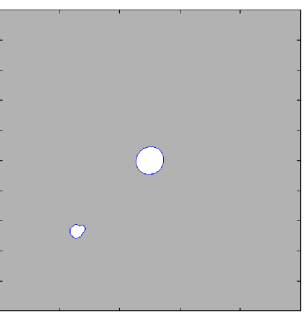

itération 111

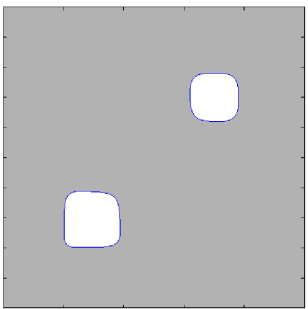

itération 40
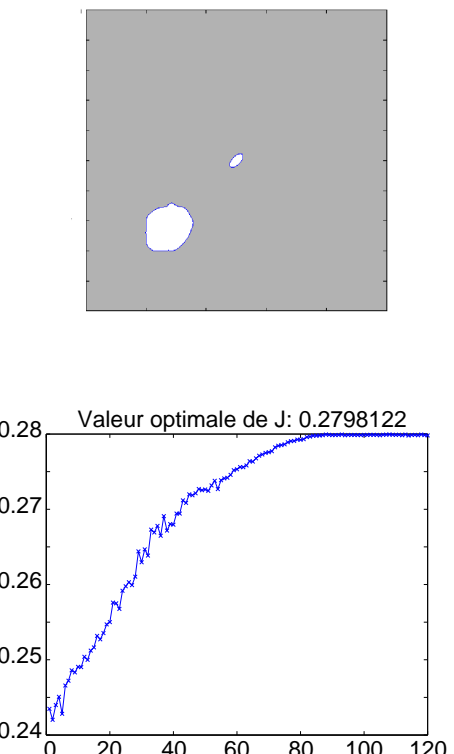

Figure 3. Evolution des domaines et fonctionnelle $J$ pour le cas non-linéaire avec utilisation de la dérivée topologique.

[10] S.A. Nazarov, J. Sokolowski, The topological derivative of the Dirichlet integral due to formation of a thin ligament Siberian Math. J. March - April 2004, Volume 45, Issue 2, 341-355.

[11] S. Osher, R. Fedkiw, Level set methods and dynamic implicit surfaces. Springer, 2004.

[12] J. Sethian, Level set methods. Cambridge University Press, 1996.

[13] J. Sokоєowsкi, A. Żochowski, On the topological derivative in shape optimization, SIAM Journal on Control and Optimization. 37 (1999), no. 4, pp. 1251-1272.

[14] J. SokoŁowski, A. Żochowski, Optimality conditions for simultaneous topology and shape optimization, SIAM Journal on Control and Optimization, 42 (2003), no. 4 , pp. 1198-1221.

[15] J. SokoŁowski, A. Żochowski, Topological derivatives for contact problems, Numer. Math., 102 ( 2005), no. 1, pp. 145-179.

[16] J. SokoŁowski, A. Żochowski Topological derivatives for obstacle problems Les prépublications de l'Institut Élie Cartan No. $12 / 2005$.

[17] J. Sokolowski, J.-P. Zolesio, Introduction to shape optimization, vol. 16 of Springer Series in Computationnal Mathematics, Springer Verlag,Berlin, 1992. 